\title{
El uso de las canciones para mejorar la habilidad de escucha en inglés ${ }^{1}$
}

\author{
Sergio Alonso Lopera Medina*
}

\begin{abstract}
Resumen
Este artículo aborda el efecto de uso de las canciones en la habilidad de escucha a un grupo que estudia inglés como lengua extranjera. Como método de investigación se aplicó el estudio de caso y se usaron tres instrumentos para la recolección de la información: el diario del profesor, un cuestionario y un grupo focal. Los resultados indican que las actividades de pre-escucha, mientras escucba y post-escucha ayudan no solo al progreso de comprensión de escucha sino también al incremento del vocabulario y a la motivación. Sin embargo, los estudiantes muestran un grado de ansiedad por entender todas las palabras en las canciones.
\end{abstract}

Palabras clave: canciones, actividades de escucha, enseñanza inglés

\section{The use of songs to improve english listening skills}

\section{ABSTRACT}

This article discusses the effects of using songs for developing listening skills in a group of people studying English as a foreign language. This case study was applied as a research method which used three instruments for data collection: the teacher diary, a questionnaire and a focus group. The results indicate that the activities of pre-listening, listening and post-listening not only help to develop listening comprehension but also to increase vocabulary and motivation. However, students show some level of anxiety in trying to understand every word in the songs.

Key words: songs, listening activities, English teaching

I Este artículo surge de una investigación de enseñanza de aula aplicada por la motivación de un docente del idioma inglés.

* Miembro del grupo de investigación EALE. Estudiante de Doctorado en Lingüística, profesor tiempo completo Escuela de Idiomas, Universidad de Antioquia, UdeA, calle 70 No. 52-2I,

Medellín, Colombia.slopera@idiomas.udea.edu.co 


\section{Introducción}

El inglés se ha convertido en una herramienta esencial a nivel académico y laboral para las personas que desean escalar profesionalmente en sus contextos. Por esta razón, universidades, colegios e institutos ofrecen una gran variedad de cursos que capacitan a los estudiantes en las cuatro habilidades (escuchar, hablar, leer y escribir).

Una de las debilidades comunes que se encuentran en los estudiantes de lenguas extranjeras es la precisión a nivel de escucha. Las razones que sustentan esta deficiencia varían: la poca atención que se hace en las habilidades de escucha, la práctica de escucha no se hace consistentemente dentro del aula de clase, y la falta de motivación para que los estudiantes practiquen (Lopera, 2003).

Para el mejoramiento a nivel de escucha, se hace necesario entonces aplicar una propuesta metodológica que incluya un trabajo sistemático de escucha dentro del aula de clase. Este artículo explora el efecto que tienen las actividades de escucha aplicadas con canciones a un grupo que estudia inglés como lengua extrajera. El artículo inicia con el marco teórico y le continúa la metodología de investigación. Luego se presenta la intervención pedagógica y los resultados de la investigación. Finalmente se brindan las conclusiones.

\section{Marco teórico}

\subsection{Ventajas y criterio de las canciones}

Duarte, Tinjacá y Carrero (20I2), Morales (2008) y Lopera (2003) sostienen que el uso de las canciones es beneficioso para el aprendizaje del idioma. Entre las ventajas del uso de las canciones se encuentran:

- Las canciones ofrecen uso real (auténtico) del idioma.

- Las canciones brindan un ambiente diferente de aprendizaje y conlleva a la motivación.

- Las canciones sirven como práctica de comprensión de escucha.

- También, las canciones se usan como medio para hacer actividades de lectura, escritura y conversación. 
- Del mismo modo, se pueden hacer ejercicios de gramática y vocabulario.

Existen diferentes criterios e ideas sobre el uso de las canciones para mejorar la escucha. Una de ellas tiene que ver con la selección de las canciones para que vaya en pro del aprendizaje y motivación. Otro criterio se relaciona con la temática del programa para que se cumplan los objetivos. También, se deben mirar las necesidades de los estudiantes, el nivel de lengua que tienen y los gustos de los estudiantes.

Con relación a la temática y las necesidades de los estudiantes, si el programa involucra reconocimiento de escucha del pasado regular ed, es importante entonces seleccionar una canción que haga una descripción en pasado. Si el nivel de lengua de los estudiantes es bajo, se deben realizar ejercicios simples de reconocimiento y completación. Además, se debe guiar a los estudiantes con ejercicios de pre-escucha, durante escucha y post-escucha. Finalmente, se puede incluir a los estudiantes en la selección de las canciones mediante un cuestionario o un listado de canciones para que ellos tengan las opciones de escoger (Lopera, 2003).

\subsection{Desventajas de las canciones}

Existen dos desventajas cuando se usan las canciones en los contextos de enseñanza. La primera tiene que ver con el cambio dramático de las canciones. Los "hits" cambian en pocas semanas o meses y esto hace que la gente olvide la gran mayoría de los éxitos musicales; sólo algunas canciones permanecen en la mente de las personas y se pueden convertir en clásicos (Murphey, I992). El término clásico se usa para enmarcar temas musicales que no deberían ser desechados una vez que se enseñan, sino que se pueden usar en otros momentos. De esta manera los estudiantes continuarán escuchándolos, entendiéndolos y disfrutándolos.

La segunda desventaja tiene que ver con las actitudes que tienen los estudiantes hacia la música. Algunos profesores y estudiantes pueden pensar que se pierde tiempo cuando se trabaja con canciones. Como consecuencia, se podría mirar que el trabajo con las canciones no se 
articula al programa de la asignatura y simplemente es una actividad para cambiar la atmósfera de clase. También, a algunos estudiantes simplemente no les gustan las canciones en inglés debido a que algunas de ellas han pasado de moda, no entienden el mensaje o prefieren otro tipo de música.

\subsection{El trabajo de escucha en las canciones}

El trabajo de escucha debe incluir los procesos de orden superior y orden inferior. Los primeros se basan en los procesos de macro conceptos que involucran la activación del conocimiento esquemático y el conocimiento contextual (Celce-Murcia, 1995; Carrell, I983, Cook, 1990). En el conocimiento esquemático se integra el conocimiento previo (background knowledge) y el conocimiento socio-cultural; también, el esquema formal que tiene que ver con la organización retórica del discurso. Con relación al conocimiento contextual, este involucra no sólo la comprensión de escucha de una situación específica (el tema, el contexto) sino también el entendimiento del discurso que está en marcha (Ur, I986).

Los procesos de orden inferior de escucha involucran el conocimiento lingüístico del oyente para que distinga e interprete los sonidos que forman las palabras o las oraciones. Para los hablantes nativos este proceso es automático, pero para los estudiantes de lenguas extranjeras este proceso causa dificultades en la comprensión auditiva si no se hace un trabajo de preparación.

Una de las formas de preparación de escucha es a través de la combinación de procesos de orden superior y procesos de orden inferior. Esta combinación le permite al oyente tener elementos lingüísticos y contextuales que le ayudarán a obtener el mensaje de una manera más efectiva en el material de audio. Una de las formas de unir ambos procesos es a través del diseño de actividades "tasks". Nunan (1989) sostiene que las actividades son una herramienta de trabajo en las aulas de clase ya que permiten a los estudiantes manipular, comprender, interactuar y producir en el idioma que se enseña; además, con el uso de las actividades los estudiantes prestan mucha mayor atención al significado que a la forma. 
Unas de las recomendaciones para trabajar las canciones es a través de las actividades de pre-escucha, durante escucha y post-escucha (Ur, I986). El objetivo de las actividades de pre-escucha es preparar al oyente a que active su conocimiento previo. El rol del profesor es de vital importancia a nivel de diseño de actividades ya que él debe hacer una transición entre lo que los estudiantes saben de una temática determinada y lo que necesitan saber de acuerdo a la canción que se va a usar.

Las actividades de durante escucha tienen como objetivo interactuar inmediatamente con la canción. En esta fase, las actividades pueden apuntar a que los oyentes respondan, completen, marquen opciones que aparecen en la canción. Finalmente, las actividades de post-escucha tienen como objetivo verificar y expandir el conocimiento que se ha adquirido con las actividades previas.

\section{Metodología de investigación}

Esta investigación se enmarca en el enfoque cualitativo que favorece el estudio de caso (Dooley, 2002; Yin, 1994). Este enfoque se utiliza para descubrir, refinar y probar hipótesis y utiliza métodos tales como la observación y la descripción, cuyo fin es reconstruir una realidad en un grupo social determinado (Hernández, Fernández y Baptista, 2003). También, esta investigación se clasifica como estudio descriptivo ya que su objetivo es explicar y describir las condiciones y efectos del uso de las canciones para mejorar la habilidad de escucha en un grupo de estudiantes de inglés como lengua extranjera.

\subsection{Preguntas de investigación}

Las preguntas de investigación que guiaron el estudio fueron:

- ¿Cuál es el efecto a nivel de escucha con uso de las canciones a través de actividades a un grupo que estudia inglés como lengua extranjera?

- ¿Cómo se percibe el trabajo con las canciones? 


\subsection{Instrumentos de recolección de información}

Tres instrumentos se utilizaron para recoger la información: el diario del profesor, un cuestionario y un grupo focal. Cada instrumento se describe a continuación:

Diario del profesor: el profesor escribía todas sus observaciones y reflexiones acerca del uso de las canciones con sus estudiantes. El objetivo de este instrumento era analizar la percepción y análisis del profesor acerca de su intervención pedagógica (Jeffrey y Hadley, 2002).

Cuestionario: se aplicó un cuestionario en la mitad de la intervención pedagógica. Este cuestionario tenía como fin indagar a los estudiantes sobre las actividades que se realizaron con las canciones (Ver ANEXO I).

Grupo focal: en la clase final del curso el profesor hizo una sesión de grupo focal para discutir las experiencias académicas con relación a las actividades con las canciones (Dendinger, 2000).

\subsection{Participantes}

Las personas que participaron en la investigación eran estudiantes del nivel IV del programa Multilingua ${ }^{2}$, con un nivel de inglés de referencia BI, según la clasificación del Marco Común Europeo de referencia para las lenguas. Los participantes eran estudiantes de pregrado de diferentes áreas (ingeniería, educación, química, biología, y derecho) y sus edades oscilaban entre 20 y 25 años de edad. II estudiantes participaron, 8 mujeres y 3 hombres; el curso inició en febrero y finalizó en junio del 2008; se dictó dos veces por semana en sesiones de clase de 2 horas cada día, para un total de 80 horas. El texto guía era Interchange, Third Edition, book III.

2 Multilingua es un programa gratuito que ofrece 8 idiomas (inglés, francés, italiano, portugués, chino, turco, japonés y alemán) a los mejores estudiantes de pregrado de la Universidad de Antioquia. Se brindan 5 niveles de 80 horas en cada idioma. 


\section{Intervención pedagógica (procedimiento)}

El profesor utilizó 7 canciones en su intervención pedagógica (Another day in paradise - Phil Collins; Imagine - the Beatles; Fatber and sonCat Stevens; Earth song - Michael Jackson; Yellow, Coldplay; You're beautiful - James Blunt; The reason - Hoobastank). El docente escogió y diseñó las 4 primeras canciones con el criterio de la temática del curso (problemas sociales) y por su claridad a nivel de escucha. Las otras 3 canciones las escogieron los estudiantes y su criterio era a nivel de motivación: "les gustaban mucho”. El docente diseñó a cada canción actividades de pre-escucha, durante escucha y post-escucha, las cuales contenían procesos de orden superior y procesos de orden inferior. El docente utilizaba 30 minutos aproximadamente de cada clase para el desarrollo de las canciones y tomaba alrededor de 2 semanas para finalizar todas las actividades de cada canción.

El docente iniciaba con las actividades de pre-escucha. En esta fase el docente realizaba actividades que preparaban a los estudiantes para las secciones específicas de escucha de la canción y de esta forma activaba el conocimiento previo de los oyentes. En esta sección el docente diseñó ejercicios de predicción y brindaba algunos apartes de expresiones que aparecerían luego en la canción (Ver diseño de una canción en el ANEXO 2). Con relación a la fase de durante escucha el docente diseñó actividades de comprensión auditiva tales como verdadero o falso, completación, marque la opción correcta, organice las oraciones en su forma correcta, entre otras. Por último, la fase final incluía actividades de discusiones del tema de la canción, de aplicación en la vida diaria, de brindar puntos de vista, que se enmarcaron en la fase de post-escucha.

Con relación al trabajo de los estudiantes con las actividades, el profesor elegía estudiantes específicos para que le brindaran las respuestas a determinados ejercicios; las actividades de pre-escucha y post-escucha las asignaba en grupos, y las actividades de durante escucha las asignaba individualmente. Con relación a las actividades en grupo, el profesor llamaba al azar a los estudiantes para que brindaran las respuestas a las actividades. Finalmente, el docente repetía segmentos de las canciones cuando los estudiantes tenían dificultades al escuchar. 


\section{Resultados}

Para analizar la información, el investigador leyó las entradas del diario, el cuestionario y las notas del grupo focal. Luego codificó la información para obtener categorías y triangular la información (Freeman, 1998). Los resultados son los siguientes:

\subsection{Progreso en la comprensión de escucha}

Uno de los hallazgos importantes es el progreso a nivel de comprensión de escucha. Al comienzo de la investigación el docente tenía que repetir muchas veces una canción para las actividades de mientras escucha, pero al final se hace la observación que disminuyó notablemente la repetición. En una de sus entradas del diario él reporta:

Una de las observaciones que tengo que bacer está relacionada con el progreso en escucha en los estudiantes. Al comienzo tenía que repetir basta 6 veces un trozo de la canción para que los estudiantes pudieran bacer las actividades de escucha. Abora al final solo repito una o dos veces.

Los estudiantes también reportaron que su comprensión auditiva en el idioma extranjero inglés había mejorado ya que entienden con mayor facilidad las canciones. Uno de los estudiantes reportó en el grupo focal:

...y creo que be progresado notablemente debido a que no tengo la necesidad de repetir tantas veces un trozo de la canción para entender la canción...

En el cuestionario, los estudiantes también reportaron que habían progresado a nivel de escucha debido a que entienden más rápidamente las canciones:

Con los ejercicios entiendo más rápido las canciones.

Creo que be mejorado mi escucha debido a que puedo entender con algún grado de facilidad los ejercicios.

Sin embargo, el investigador miró con cuidado este elemento y concluyó que los estudiantes progresaron su nivel de escucha y que se rebajó el número de repeticiones por dos razones: la primera tiene que ver con el contacto con las actividades, y el usarlas eficazmente. 
Los estudiantes aprendían o codificaban las expresiones o vocabulario que se trabaja en la fase de pre-escucha e intentaban encontrarlas en la segunda fase (mientras escucba). Esto les ayudó notablemente a entender algunos apartes de las canciones. La segunda razón tiene que ver con la familiaridad con las canciones que ellos escogieron por su propia cuenta. Estas canciones se utilizaron al final de la intervención pedagógica y esto pudo incidir en el desarrollo de la comprensión auditiva. No obstante, algunos de los estudiantes expresaron en el grupo focal que solo se sabían el coro de las canciones que seleccionaron:

... no, sólo algunas palabras o expresiones, especialmente el coro...

... sí, yo sólo sabía pronunciar el coro pero no sabía qué decía, ja, ja, ja...

Por otro lado, los estudiantes tuvieron dificultad con el reconocimiento auditivo de algunas palabras. Por ejemplo, en la expresión "he pretends he can't hear her" de la canción "Anotber Day in Paradise" los estudiantes tuvieron dificultad de reconocimiento del pronombre objeto "her". El docente tuvo que repetir varias veces y pronunciarla él mismo para que los estudiantes la reconocieran. Otras palabras o expresiones que les brindaron dificultad fueron: I'll, harder, I'd, thought up, below us, we've, take it all away, I jumped across. El docente observó con cuidado los elementos de dificultad en la comprensión auditiva y concluyó que las contracciones, reducciones, sufijos y los pronombres eran los elementos lingüísticos que presentaban mayor dificultad.

\subsection{Reconocimiento e incremento del vocabulario}

Otro importante hallazgo tiene que ver con el vocabulario. Tanto el profesor como los estudiantes observaron que habían progresado con el vocabulario ya que los participantes escuchaban y reconocían con mayor facilidad palabras o expresiones en algunas de las canciones. La fase de pre-escucha jugó un papel importante para el reconocimiento auditivo. De hecho, el profesor argumentó en su diario:

... los estudiantes empiezan a reconocer auditivamente con mayor facilidad algunas palabras y expresiones que se presentan en las canciones. En un par 
de ocasiones presenté las canciones sin bacer actividades de pre-escucha y los estudiantes reconocieron pocas palabras o expresiones. Sin embargo, cuando se bacian explíitos los ejercicios de pre-escucba los estudiantes reconocían con mayor facilidad el vocabulario o expresiones.

En el grupo focal los estudiantes admitieron que habían progresado con el vocabulario; uno de ellos comentó:

Una de las cosas buenas de este trabajo con las canciones es que uno aprende más vocabulario...

\subsection{Motivación}

La motivación juega un papel importante en el aprendizaje de los idiomas (Dörnyei, 1994). Los estudiantes mostraron motivación con el hecho de trabajar la competencia auditiva con el uso de las canciones. El profesor expresó en su diario que este trabajo creaba un ambiente agradable en el salón de clase:

... también noto que los estudiantes toman una posición muy positiva y que bay un buen ambiente en el salón de clase. De becho, noto que los estudiantes se ríen, comparten elementos personales, comentan chistes como "qué sordo soy yo"...

Sin embargo, el profesor notó un grado de ansiedad al comienzo del trabajo con las canciones debido a que los estudiantes querían entender auditivamente cada palabra. Para ellos una comprensión exitosa es entender cada palabra de la canción. No obstante, el profesor insistió sobre el proceso natural de los estudiantes de lenguas extranjeras a no entender cada palabra pronunciada; de hecho él argumento en su diario:

... debo vender la idea de que no se entiende completamente la canción al inicio ya que es un proceso de comprensión auditiva y las actividades apuntan a esto.

Finalmente el profesor observó que esta ansiedad desapareció con las últimas canciones:

... y noto abora al final que la ansiedad de entender la canción por completo mermó considerablemente. 
Con relación a la percepción del trabajo con las canciones, hubo un consenso general positivo acerca de las actividades con las canciones. 8 estudiantes reportaron que las actividades fueron excelentes y 3 que eran buenas. En cuanto al número de actividades realizadas en clase, 7 estudiantes reportaron que apenas fueron, 3 pocas, I muchas. Finalmente, todos los estudiantes reportaron que el tiempo utilizado en clase con el trabajo con las canciones fue apropiado.

Finalmente, los estudiantes hicieron un par de sugerencias u observaciones importantes con el trabajo con las canciones: la primera es con relación a la selección de las canciones. Los estudiantes quieren trabajar con canciones "nuevas" (los hits de último momento) y con clásicos. La segunda sugerencia gira en torno a que los profesores de los cursos de inglés deben trabajar las canciones con este tipo de actividades.

\section{Conclusiones}

El uso de las canciones con actividades diseñadas puede ayudar a desarrollar la habilidad de escucha en una lengua extranjera. Con relación a esta investigación, la combinación de actividades de pre-escucha, mientras escucha y post-escucha ayudaron no solamente al progreso de comprensión de escucha sino también al incremento del vocabulario. Además, los estudiantes mostraron motivación con el hecho de trabajar la competencia auditiva con el uso de las canciones. También, la percepción que tuvieron los participantes sobre la calidad, el número y el tiempo de las actividades fue positiva.

\subsection{Discusión}

Surgieron dos elementos importantes para tener en cuenta con la enseñanza con las canciones: el primero tiene que ver con el grado de ansiedad de los estudiantes. La idea de tolerar la canción sin entenderla completamente es vital en este trabajo. Es importante que el docente concientice a los estudiantes a que el desarrollo de la habilidad de escucha es progresiva y se hace a través de las actividades. La segunda tiene que ver con la inclusión de los estudiantes para el criterio de selección de canciones. Se sugiere tener en cuenta a los 
estudiantes para la elección de una canción ya que esta inclusión podría traer efectos positivos para el aprendizaje.

Finalmente, los profesores de inglés deben tener en cuenta el formato de pre, mientras y post para ser usado no sólo en la habilidad de escucha sino también en la lectura y escritura. Esto traería también efectos positivos en la enseñanza en las diferentes habilidades.

\section{Referencias bibliográficas}

Carrell, P. (1983). Three components of background knowledge in reading comprehension. Language Learning, 33 (2), I83-203.

Celce-Murcia, M. (1995). Discourse analysis and the teaching of listening. En: Cook, G., and Seidlhofer, B. Principles and practice in applied linguistics. Oxford: Oxford University Press.

Cook, G. (1990). Two approaches to developing discourse skills. Discourse. Oxford: Oxford University Press.

Cresswell, J.W. (2007). Qualitative inquiry and research design. Choosing among five approaches. Thousand Oaks: CA. SAGE Publications.

Dendinger, M. (2000). How to organize a focus group. Meetings and conventions. Recuperado el 25 de mayo de 2012 de http://www. meetings-conventions.com/articles/how-to-organize-a-focus-group/ cI0136.aspx

Dooley, L. (2002). Case study research and theory building. Advances in Developing Human Resources, 4 (3), 335-354.

Dörnyei, Z. (1994). Motivation and motivating in the foreign language classroom. Modern Language Journal, 78, 273-284.

Duarte, M.; Tinjacá, L.; Carrero, M. (2012). Using songs to encourage sixth graders to develop English speaking skills. PROFILE, I4 (I), I I-28.

Freeman, D. (I998). Doing teacher research: From inquiry to understanding. Boston, MA: Newbury House.

Hernández, R.; Fernández, C. y Baptista, P. (2003). Metodología de la investigación. México: McGraw-Hill Interamericana, tercera edición.

Jeffrey, D.; Hadley, G. (2002). Balancing Intuition with Insight: Reflective Teaching through Diary Studies. The Language Teacher Online, 26 (5).

Lopera, S. (2003). Useful ideas when taking songs to a class. IKALA. 8 (I4), I35-I52. 
Morales, C. (2008). Using rock music as a teaching-learning tool. PROFILE, 9, I63-I80.

Murphey, T. (I992). Music and song. Oxford: Oxford University Press.

Nunan, D. (1988). Syllabus design. Oxford: Oxford University Press.

Nunan, D. (1989). Designing tasks for the communicative classroom. Cambridge: Cambridge University Press.

Ur, P. (1986). Teaching listening comprehension. Cambridge: Cambridge University Press.

Yin, R. K. (2003). Case study research. Design and Methods. ( $3^{\text {rd }}$ edition). Thousand Oaks: CA. SAGE Publications. 